\title{
Design of Attitude Control System for UAV Based on Feedback Linearization and Adaptive Control
}

\author{
Wenya Zhou, ${ }^{1,2}$ Kuilong Yin, ${ }^{2}$ Rui Wang, ${ }^{1,2}$ and Yue-E Wang ${ }^{3}$ \\ ${ }^{1}$ State Key Laboratory of Structural Analysis for Industrial Equipment, Dalian University of Technology, Dalian 116023, China \\ ${ }^{2}$ School of Aeronautics and Astronautics, Dalian University of Technology, Dalian 116023, China \\ ${ }^{3}$ College of Mathematics and Information Science, Shanxi Normal University, Xian 710062, China
}

Correspondence should be addressed to Wenya Zhou; zwy@dlut.edu.cn

Received 6 January 2014; Accepted 9 February 2014; Published 20 March 2014

Academic Editor: Huaicheng Yan

Copyright (C) 2014 Wenya Zhou et al. This is an open access article distributed under the Creative Commons Attribution License, which permits unrestricted use, distribution, and reproduction in any medium, provided the original work is properly cited.

\begin{abstract}
Attitude dynamic model of unmanned aerial vehicles (UAVs) is multi-input multioutput (MIMO), strong coupling, and nonlinear. Model uncertainties and external gust disturbances should be considered during designing the attitude control system for UAVs. In this paper, feedback linearization and model reference adaptive control (MRAC) are integrated to design the attitude control system for a fixed wing UAV. First of all, the complicated attitude dynamic model is decoupled into three single-input single-output (SISO) channels by input-output feedback linearization. Secondly, the reference models are determined, respectively, according to the performance indexes of each channel. Subsequently, the adaptive control law is obtained using MRAC theory. In order to demonstrate the performance of attitude control system, the adaptive control law and the proportional-integral-derivative (PID) control law are, respectively, used in the coupling nonlinear simulation model. Simulation results indicate that the system performance indexes including maximum overshoot, settling time ( $2 \%$ error range), and rise time obtained by MRAC are better than those by PID. Moreover, MRAC system has stronger robustness with respect to the model uncertainties and gust disturbance.
\end{abstract}

\section{Introduction}

The applications of unmanned aerial vehicles (UAVs) have dramatically extended in both military and civilian fields around the world in the last twenty years. UAVs are used currently in all branches of military ranging from investigation, monitoring, intelligence gathering, and battlefield damage assessment to force support. Civilian applications include remote sensing, transport, exploration, and scientific research. Because of the diversified mission in the aviation field, UAVs play a more and more important role.

The attitude dynamic model of UAVs is nonlinear and three attitude channels are coupled. Nonlinearity and coupling dynamic characteristics will become more disturbing under flight condition with big angle of attack so that more obstacles will be brought during the design of attitude control system for UAVs.

In recent years, some advanced control theories are gradually introduced into the design of attitude control system for UAVs with the development of computer technology [117]. In [1], the output feedback control method was used to design the attitude control system for UAV. An observer was designed to estimate the states online. In [2], in order to provide a basis for comparison with more sophisticated nonlinear designs, a PID controller with feedforward gravity compensation was derived using a small helicopter model and tested experimentally. In [3], a roll-channel fractional order proportional integral $\left(\mathrm{PI}^{\lambda}\right)$ flight controller for a small fixed-wing UAV was designed and time domain system identification methods are used to obtain the roll-channel model. In [4], a second-order sliding structure with a secondorder sliding mode including a high-order sliding mode observer for the estimation of the uncertain sliding surfaces was selected to develop an integrated guidance and autopilot scheme. In [5], the fuzzy sliding mode control based on the multiobjective genetic algorithm was proposed to design the altitude autopilot of UAV. In [6], the attitude tracking system was designed for a small quad rotor UAV through model 
reference adaptive control method. In [7], to control the position of UAV in three dimensions, altitude and longitudelatitude location, an adaptive neurofuzzy inference system was developed by adjusting the pitch angle, the roll angle, and the throttle position. In [8], an $L_{1}$ adaptive controller as autopilot inner loop controller candidate was designed and tested based on piecewise constant adaptive laws. Navigation outer loop parameters are regulated via PID control. The main contribution of this study is to demonstrate that the proposed control design can stabilize the nonlinear system. In [9], an altitude hold mode autopilot for UAV which is nonminimum phase was designed by combination of classic controller as the principal section of autopilot and the fuzzy logic controller to increase the robustness. The multiobjective genetic algorithm is used to mechanize the optimal determination of fuzzy logic controller parameters based on an efficient cost function that comprises undershoot, overshoot, rise time, settling time, steady state error, and stability. In $[10,11]$, a novel intelligent control strategy based on a brain emotional learning (BEL) algorithm was investigated in the application of attitude control of UAV. Time-delay phenomenon and sensor saturation are very common in practical engineering control and is frequently a source of instability and performance deterioration [18-20]. Taking time delay into consideration, the influence of time delay on the stability of the low-altitude and low-speed small Unmanned Aircraft Systems (UAS) flight control system had been analyzed [21]. There are still some other methods [22, 23]; no details will be listed here.

The design method based on characteristic points can obtain multiple control gains. To realize the gain scheduling, look-up table is one common way applied in practice. Actually, the gains between characteristic points do not exist but can be obtained only by interpolation method. It is hard to ensure the control satisfaction between characteristic points [24]. As for sliding control method, it is difficult to select the intermediate control variables for partial derivatives of a sliding surface. As for the intelligent control method, though the stability of control system can be verified, the algorithm is too complicated and it is unable to guarantee the control timeliness in application. In a word, the attitude control system design of UAVs is an annoying task. Multi-input multioutput (MIMO), nonlinearity, and coupling dynamic characteristics will cause more difficulties during the design of attitude control system. In addition, model uncertainties and external disturbances should be taken into account also.

Feedback linearization method can be used to realize linearization and decoupling of a complicated model. Model reference adaptive control (MRAC) system can suppress model uncertainties and has stronger robustness with respect to gust disturbances. With these considerations, feedback linearization method and MRAC method are integrated to design the attitude control system for a fixed wing UAV. As far as we know, there is only few research in which the above two methods are integrated. Moreover, this design principle is simple and the control performance is superior. The maximum overshoot, settling time, and rise time of the system can satisfy the desired indexes, and the system has strong robustness with respect to the uncertainties of aerodynamic parameters variation and gust disturbance.

This paper is organized as follows. firstly, the complicated attitude dynamic model is decoupled into three independent channels by feedback linearization method; secondly, according to the control performance indexes of each attitude channel, such as maximum overshoot, settling time, and rise time, reference model is established and MRAC is used to design the adaptive control law; thirdly, the control performance comparison between MRAC and PID control is given; finally, conclusions are presented.

\section{Attitude Dynamic Model of UAV}

The origin $O$ of UAV body coordinate system $O x_{b} y_{b} z_{b}$ is located at mass center. Axis $x_{b}$ coincides with aircraft longitudinal axis and points to the nose. Axis $y_{b}$ is perpendicular to aircraft longitudinal symmetric plane and points to the right side. Axis $z_{b}$ is defined following the right-hand rule. The dynamic models of three attitude channels including roll, pitch, and yaw are given as follows:

$$
\begin{gathered}
\dot{\phi}=p+\tan \theta(r \cos \phi+q \sin \phi), \\
\dot{\theta}=q \cos \phi-r \sin \phi \\
\dot{\psi}=\frac{r \cos \phi+q \sin \phi}{\cos \theta}, \\
\dot{p}=\left[I_{z} L+I_{x z}\left(N+\left(I_{x}+I_{z}-I_{y}\right) p q\right)\right. \\
\left.-q r\left(I_{z}^{2}+I_{x z}^{2}-I_{y} I_{z}\right)\right] \times\left(I_{x} I_{z}-I_{x z}^{2}\right)^{-1} \\
\dot{q}=\frac{M-p r\left(I_{x}-I_{z}\right)-I_{x z}\left(p^{2}-r^{2}\right)}{I_{y}} \\
\dot{r}=\frac{\left[I_{x z} L+I_{x} N+p q\left(I_{x}^{2}+I_{x z}^{2}-I_{y} I_{x}\right)+q r I_{x z}\left(I_{y}-I_{x}-I_{z}\right)\right]}{\left(I_{x} I_{z}-I_{x z}^{2}\right)} .
\end{gathered}
$$

The models describe the behavior of aircraft following control input, where $\phi, \theta$, and $\psi$ represent roll angle, pitch angle, and yaw angle, respectively; $p, q$, and $r$ represent the angle velocity components on body axis $x_{b}, y_{b}$, and $z_{b} ; I_{x}, I_{y}$, and $I_{z}$ represent the inertia moment of body axis; $I_{x z}$ denotes the inertia product against axis $O x_{b}$ and $O z_{b} ; L, M$, and $N$ represent the resultant moment components on body axis $x_{b}$, $y_{b}$, and $z_{b}$, and

$$
\begin{aligned}
L=\frac{1}{2} \rho V^{2} S_{w} b & \left(C_{l \beta} \beta+C_{l \dot{\beta}} \dot{\beta}+C_{l \delta a} \delta_{a}\right. \\
& \left.+C_{l \delta r} \delta_{r}+C_{l r} r+C_{l p} p\right), \\
M=\frac{1}{2} \rho V^{2} S_{w} c( & C_{m 0}+C_{m \alpha} \alpha+C_{m \delta e} \delta_{e} \\
& \left.+C \dot{\alpha}+C_{m q} q\right),
\end{aligned}
$$




$$
\begin{aligned}
N=\frac{1}{2} \rho V^{2} S_{w} b( & C_{n \beta} \beta+C_{n \dot{\beta}} \dot{\beta}+C_{n \delta a} \delta_{a} \\
& \left.+C_{n \delta r} \delta_{r}+C_{n r} r+C_{n p} p\right),
\end{aligned}
$$

where $\rho$ is the atmosphere density relative to height; $V$ is airspeed of UAV; $S_{w}, b$, and $c$ represent wing area, span, and mean aerodynamic chord, respectively; $\beta$ and $\alpha$ represent sideslip angle and attack angle, respectively. $\delta a, \delta e$, and $\delta r$ represent the deflection angle of aileron, elevator, and rudder, respectively; $C$ represents the aerodynamic moment coefficient and its subscript is composed of corresponding moments and variables, where $C_{m 0}$ represents the aerodynamic moment coefficient at $0^{\circ}$ attack angle.

It is obvious that attitude dynamic model of UAV is nonlinear and there are strong coupling among three channels. Substitute the aerodynamic moment equations (7) into attitude dynamic model equations (4) (6) and rewrite them as follows:

$$
\begin{gathered}
\dot{\mathbf{x}}=\mathbf{f}(\mathbf{x})+\mathbf{g u}, \\
\mathbf{y}=\mathbf{h}(\mathbf{x}),
\end{gathered}
$$

where

$$
\begin{aligned}
& \mathbf{x}=\left[\begin{array}{llllll}
p & q & r & \phi & \theta & \psi
\end{array}\right]^{T}, \\
& \mathbf{u}=\left[\begin{array}{lll}
\delta a & \delta e & \delta r
\end{array}\right]^{T}, \\
& \mathbf{h}(\mathbf{x})=\left[\begin{array}{lll}
\phi & \theta & \psi
\end{array}\right]^{T}, \\
& \mathbf{f}(\mathbf{x})=\left[\begin{array}{llllll}
f_{1} & f_{2} & f_{3} & f_{4} & f_{5} & f_{6}
\end{array}\right]^{T}, \\
& f_{1}=\left[2 p q I_{z x}\left(I_{z}+I_{x}-I_{y}\right)+2 q r\left(I_{y} I_{z}-I_{z}^{2}-I_{z x}^{2}\right)\right. \\
& +I_{z} \rho V^{2} S_{w} b\left(C_{l \beta} \beta+C_{l \dot{\beta}} \dot{\beta}+C_{l r} r+C_{l p} p\right) \\
& \left.+I_{z x} \rho v^{2} S_{w} b\left(C_{n \beta} \beta+C_{n \dot{\beta}} \dot{\beta}+C_{n r} r+C_{n p} p\right)\right] \\
& \times\left(2 I_{x} I_{z}-2 I_{z x}^{2}\right)^{-1} \\
& f_{2}=\left[-2 p r\left(I_{x}-I_{z}\right)-2\left(p^{2}-r^{2}\right) I_{z x}\right. \\
& \left.+\rho V^{2} S_{w} c\left(C_{m 0}+C_{m \alpha} \alpha+C_{m \dot{\alpha}} \dot{\alpha}+C_{m q} q\right)\right] \times\left(2 I_{y}\right)^{-1}, \\
& f_{3}=\left[2 p q\left(I_{z x}^{2}+I_{x}^{2}-I_{y} I_{x}\right)+2 q r\left(I_{y}-I_{z}-I_{x}\right)\right. \\
& +I_{z x} \rho V^{2} S_{w} b\left(C_{l \beta} \beta+C_{l \dot{\beta}} \dot{\beta}+C_{l r} r+C_{l p} p\right) \\
& \left.+I_{x} \rho V^{2} S_{w} b\left(C_{n \beta} \beta+C_{n \dot{\beta}} \dot{\beta}+C_{n r} r+C_{n p} p\right)\right] \\
& \times\left(2 I_{x} I_{z}-2 I_{z x}^{2}\right)^{-1} \text {, } \\
& f_{4}=p+q \sin \phi \tan \theta+r \cos \phi \tan \theta, \\
& f_{5}=q \cos \phi-r \sin \phi, \\
& f_{6}=q \sin \phi \sec \theta+r \cos \phi \sec \theta \text {, }
\end{aligned}
$$

$$
\begin{gathered}
\mathbf{g}=\frac{\rho V^{2} S_{w}}{2 I_{y}\left(I_{x} I_{z}-I_{z x}^{2}\right)}\left[\begin{array}{ccc}
g_{11} & 0 & g_{13} \\
0 & g_{22} & 0 \\
g_{31} & 0 & g_{33} \\
0 & 0 & 0 \\
0 & 0 & 0 \\
0 & 0 & 0
\end{array}\right] \\
g_{11}=I_{y} b\left(I_{z} C_{l \delta_{a}}+I_{z x} C_{n \delta_{a}}\right) \\
g_{13}=I_{y} b\left(I_{z} C_{l \delta_{r}}+I_{z x} C_{n \delta_{r}}\right) \\
g_{22}=C_{m \delta_{e}}\left(I_{x} I_{z}-I_{z x}\right) \\
g_{31}=I_{y} b\left(I_{z x} C_{l \delta_{a}}+I_{x} C_{n \delta_{a}}\right) \\
g_{33}=I_{y} b\left(I_{z x} C_{l \delta_{r}}+I_{x} C_{n \delta_{r}}\right)
\end{gathered}
$$

\section{Linearization and Decoupling of Model}

In order to obtain the SISO form of the three attitude channels, feedback linearization method is used in this paper. As for nonlinear equations (8), we can obtain the following equation according to Lie derivative:

$$
\mathbf{u}=\frac{1}{L_{g} L_{f}^{n} \mathbf{h}}\left(-L_{f}^{n} \mathbf{h}+\mathbf{v}\right),
$$

where $L_{f} h$ and $L_{g} L_{f} h$ represent Lie derivative of $\mathbf{h}$ with respect to $\mathbf{f}$ and $\mathbf{g}$. Superscript $n$ represents the derivative order. The new input $\mathbf{v}$ is $\mathbf{v}=\left[\begin{array}{lll}v_{1} & v_{2} & v_{3}\end{array}\right]^{T}$.

Let

$$
\begin{gathered}
\mathbf{Q}=L_{g} L_{f}^{n} \mathbf{h}=\left[\begin{array}{lll}
Q_{1} & Q_{2} & Q_{3}
\end{array}\right]^{T}, \\
\mathbf{P}=L_{f}^{n} \mathbf{h}=\left[\begin{array}{lll}
P_{1} & P_{2} & P_{3}
\end{array}\right]^{T} .
\end{gathered}
$$

We can get

$$
\begin{aligned}
& \mathbf{Q}_{1}^{T} \frac{1}{2} \rho V^{2} S_{w} b \\
& \cdot\left[\begin{array}{c}
\frac{\cos \phi \tan \theta\left(I_{x z} C_{l \delta a}+I_{x} C_{n \delta a}\right)+I_{z} C_{l \delta a}+I_{x z} C_{n \delta a}}{I_{x} I_{z}-I_{x z}^{2}} \\
\frac{c \sin \phi \tan \theta C_{m \delta e}}{b I_{y}} \\
\frac{\cos \phi \tan \theta\left(I_{x z} C_{l \delta r}+I_{x} C_{n \delta r}\right)+I_{z} C_{l \delta r}+I_{x z} C_{n \delta r}}{I_{x} I_{z}-I_{x z}^{2}} \\
\mathbf{Q}_{2}^{T}=\frac{1}{2} \rho V^{2} S_{w} b\left[\begin{array}{c}
\sin \phi\left(I_{x z} C_{l \delta a}+I_{x} C_{n \delta a}\right) \\
-\frac{I_{x} I_{z}-I_{x z}^{2}}{c \cos \phi C_{m \delta e}} \\
b I_{y}
\end{array}\right], \\
-\frac{\sin \phi\left(I_{x z} C_{l \delta r}+I_{x} C_{n \delta r}\right)}{I_{x} I_{z}-I_{x z}^{2}}
\end{array}\right],
\end{aligned}
$$




$$
\mathbf{Q}_{3}^{T}=\frac{1}{2} \rho V^{2} S_{w} b\left[\begin{array}{c}
\frac{\cos \phi\left(I_{x z} C_{l \delta a}+I_{x} C_{n \delta a}\right)}{\cos \theta\left(I_{x} I_{z}-I_{x z}^{2}\right)} \\
\frac{c \sin \phi C_{m \delta e}}{b \cos \theta I_{y}} \\
\frac{\cos \phi\left(I_{x z} C_{l \delta r}+I_{x} C_{n \delta r}\right)}{\cos \theta\left(I_{x} I_{z}-I_{x z}^{2}\right)}
\end{array}\right] .
$$

Expressions of $P_{1}, P_{2}$, and $P_{3}$ are more complicated and can be obtained by referring to the literature [17].

The system relative order is $n_{1}+n_{2}+n_{3}=6$ according to Lie derivative. The input and output linearization of MIMO nonlinear system is realized by the above derivation. There is no internal dynamic state in new system that asymptotic stability and tracking control can be realized. The feedback linearization diagram is shown as Figure 1.

It is visible that the nonlinear dynamic model is transformed into one equivalent linear model with state variables as follows:

$$
\overline{\mathbf{x}}=\left[\begin{array}{llllll}
\phi & \dot{\phi} & \theta & \dot{\theta} & \psi & \dot{\psi}
\end{array}\right]^{T} .
$$

State equations are rewritten in matrix form:

$$
\begin{aligned}
{\left[\begin{array}{c}
\dot{\phi} \\
\ddot{\phi} \\
\dot{\theta} \\
\ddot{\theta} \\
\dot{\psi} \\
\ddot{\psi}
\end{array}\right] } & {\left[\begin{array}{llllll}
0 & 1 & 0 & 0 & 0 & 0 \\
0 & 0 & 0 & 0 & 0 & 0 \\
0 & 0 & 0 & 1 & 0 & 0 \\
0 & 0 & 0 & 0 & 0 & 0 \\
0 & 0 & 0 & 0 & 0 & 1 \\
0 & 0 & 0 & 0 & 0 & 0
\end{array}\right]\left[\begin{array}{c}
\phi \\
\dot{\phi} \\
\theta \\
\dot{\theta} \\
\psi \\
\dot{\psi}
\end{array}\right]+\left[\begin{array}{lll}
0 & 0 & 0 \\
1 & 0 & 0 \\
0 & 0 & 0 \\
0 & 1 & 0 \\
0 & 0 & 0 \\
0 & 0 & 1
\end{array}\right] } \\
& \times\left[\begin{array}{l}
v_{1} \\
v_{2} \\
v_{3}
\end{array}\right] .
\end{aligned}
$$

Remark 1. Although new errors are not produced in the process of decoupling the dynamic equations by feedback linearization, it is impossible to describe all dynamic characteristics of attitude moment precisely, the reason for this is modeling errors and model uncertainties cannot be eliminated in dynamic model.

\section{Control Laws Design}

Aiming at the above three independent two-order systems and according to the performance indexes of attitude response, MRAC is used in this paper to design the attitude control law.

4.1. MRAC Law Design. The differential equation for each channel in (14) can be written as

$$
\ddot{y}_{p}=\bar{u}_{1} .
$$

The adaptive control law is designed by taking the pitch channel as an example. Suppose the form of control law is

$$
\bar{u}_{1}=k r+f_{0} y_{p}+f_{1} \dot{y}_{p}
$$

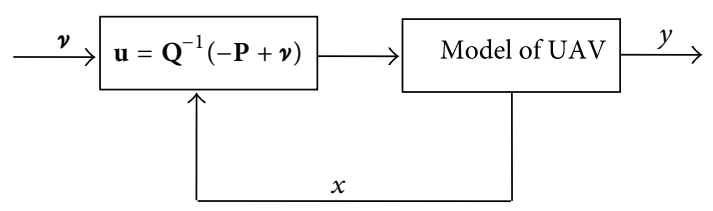

FIGURE 1: Feedback linearization diagram.

where $k$ is the feedforward gain, $r$ is the reference input, $f_{0}$ and $f_{1}$ are feedback gains. The approach of MRAC is to adjust parameters $k, f_{0}$, and $f_{1}$ so that the system output can track the output of the reference model.

Select the same order of reference model as that of pitch channel model and the differential equation is

$$
\ddot{y}_{m}+a_{1} \dot{y}_{m}+a_{0} y_{m}=b r \text {. }
$$

Coefficients $a_{0}, a_{1}$, and $b$ should be determined according to control performance indexes of pitch channel.

Consider the standard form of two-order system:

$$
\phi(s)=\frac{\omega_{n}^{2}}{s^{2}+2 \xi \omega_{n} s+\omega_{n}^{2}} .
$$

We can get

$$
\begin{gathered}
t_{s}=\frac{3.5}{\xi \omega_{n}}, \\
\sigma \%=\frac{e^{-\pi \xi}}{\sqrt{1-\xi^{2}}} \times 100 \%, \\
t_{r}=\frac{\pi-\beta}{w_{d}}, \\
w_{d}=w_{n} \sqrt{1-\xi^{2}}, \\
\xi=\cos \beta,
\end{gathered}
$$

where $\xi$ denote damping ratio; $\omega_{n}$ and $\omega_{d}$ denote natural oscillation angular frequency and damping oscillation angular frequency, respectively; $t_{s}$ and $t_{r}$ denote settling time and rise time, respectively; $\sigma \%$ denotes overshoot; set $t_{p}=5 \mathrm{~s}$, $\sigma=2 \%$; it is easy to get $\xi=0.7797, w_{n}=1.0035 \mathrm{rad} / \mathrm{s}$.

Substitute (16) into (15); the adjustable differential equation can be obtained:

$$
\ddot{y}_{p}-f_{1} \dot{y}_{p}-f_{0} y_{p}=k r \text {. }
$$

Define $e=y_{m}-y_{p}$ as the generalized error and according to (17) and (20), the generalized error equation is

$$
\begin{aligned}
\ddot{e}+a_{1} \dot{e}+a_{0} e= & -\left(a_{1}+f_{1}\right) \dot{y}_{p} \\
& -\left(a_{0}+f_{0}\right) y_{p}+(b-k) r .
\end{aligned}
$$

Let

$$
\delta_{1}=-a_{1}-f_{1}, \quad \delta_{0}=-a_{0}-f_{0}, \quad \sigma=b-k .
$$


Equation (21) can be rewritten as

$$
\ddot{e}+a_{1} \dot{e}+a_{0} e=\delta_{1} \dot{y}_{p}+\delta_{0} y_{p}+\sigma r .
$$

Define parameter error vector $\boldsymbol{\theta}$ and generalized error vector $\boldsymbol{\varepsilon}$, respectively, as

$$
\boldsymbol{\theta}=\left[\begin{array}{lll}
\delta_{0} & \delta_{1} & \sigma
\end{array}\right]^{T}, \quad \boldsymbol{\varepsilon}=\left[\begin{array}{ll}
e & \dot{e}
\end{array}\right]^{T} .
$$

Then error expression equation (23) can be written in matrix-vector form:

$$
\dot{\varepsilon}=\mathbf{A} \varepsilon+\Delta_{a}+\Delta_{b},
$$

where

$$
\begin{gathered}
\mathbf{A}=\left[\begin{array}{cc}
0 & 1 \\
-a_{0} & -a_{1}
\end{array}\right], \quad \Delta_{a}=\left[\begin{array}{c}
0 \\
\delta_{0} y_{p}+\delta_{1} \dot{y}_{p}
\end{array}\right], \\
\Delta_{b}=\left[\begin{array}{c}
0 \\
\sigma r
\end{array}\right] .
\end{gathered}
$$

Select the Lyapunov function:

$$
\bar{V}=\frac{1}{2}\left(\boldsymbol{\varepsilon}^{T} \mathbf{P} \boldsymbol{\varepsilon}+\boldsymbol{\theta}^{T} \boldsymbol{\Gamma} \boldsymbol{\theta}\right),
$$

where $\mathbf{P}$ is $2 \times 2$ positive definite symmetric matrix, $\boldsymbol{\Gamma}$ is 3 dimensional positive definite diagonal matrix:

$$
\Gamma=\operatorname{diag}\left(\begin{array}{lll}
\lambda_{0} & \lambda_{1} & \mu
\end{array}\right) .
$$

Let $\mathbf{P}=\left[\begin{array}{ll}p_{11} & p_{12} \\ p_{21} & p_{22}\end{array}\right]$ and $p_{12}=p_{21}$; we can get the derivative of $\bar{V}$ with respect to time:

$$
\begin{aligned}
\dot{\bar{V}}= & \frac{1}{2} \boldsymbol{\varepsilon}^{T}\left(\mathbf{P A}+\mathbf{A}^{T} \mathbf{P}\right) \boldsymbol{\varepsilon}+\delta_{0}\left[\lambda_{0} \dot{\delta}_{0}+\left(e p_{12}+\dot{e} p_{22}\right) y_{p}\right] \\
& +\delta_{1}\left[\lambda_{1} \dot{\delta}_{1}+\left(e p_{12}+\dot{e} p_{22}\right) \dot{y}_{p}\right] \\
& +\sigma\left[\mu \dot{\sigma}+\left(e p_{12}+\dot{e} p_{22}\right) r\right] .
\end{aligned}
$$

Select positive definite symmetric matrix $\mathbf{Q}$ and make

$$
\mathbf{P A}+\mathbf{A}^{T} \mathbf{P}=-\mathbf{Q} .
$$

Select the adaptive laws:

$$
\begin{gathered}
\dot{\delta}_{0}=-\frac{\left(e p_{12}+\dot{e} p_{22}\right) y_{p}}{\lambda_{0}}, \\
\dot{\delta}_{1}=-\frac{\left(e p_{12}+\dot{e} p_{22}\right) \dot{y}_{p}}{\lambda_{1}}, \\
\dot{\sigma}=-\frac{\left(e p_{12}+\dot{e} p_{22}\right) r}{\mu} .
\end{gathered}
$$

Obviously, $\dot{\bar{V}}$ is negative definite; therefore the closedloop system is asymptotically stable. Calculate the derivative of each equation in (24) with respect to time with considering

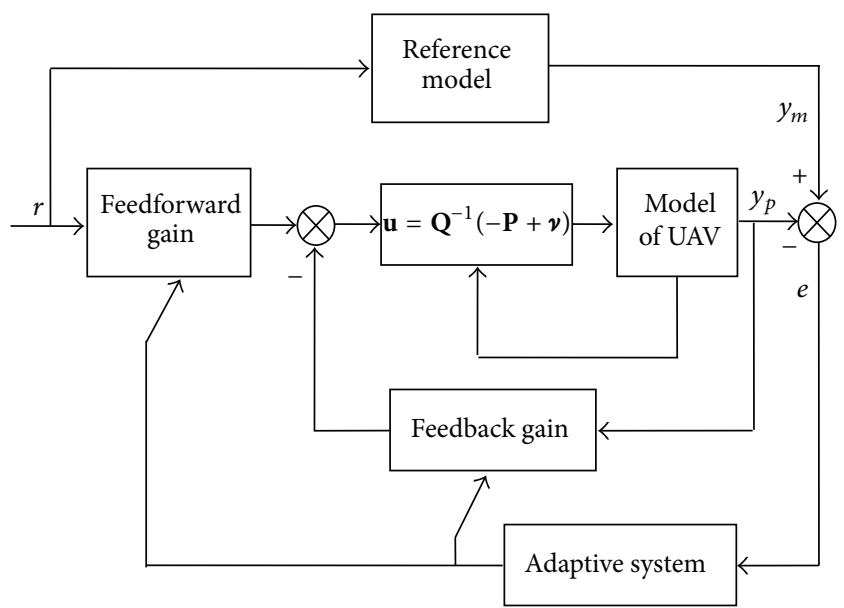

Figure 2: MRAC system diagram.

(31), the adaptive laws of feedback gains $f_{0}, f_{1}$ and feedforward gain $k$ can be obtained:

$$
\begin{aligned}
f_{0} & =\int_{0}^{t} \frac{\left(e p_{12}+\dot{e} p_{22}\right) y_{p}}{\lambda_{0}} d \tau+f_{0}(0), \\
f_{1} & =\int_{0}^{t} \frac{\left(e p_{12}+\dot{e} p_{22}\right) y_{p}}{\lambda_{1}} d \tau+f_{1}(0), \\
k & =\int_{0}^{t} \frac{\left(e p_{12}+\dot{e} p_{22}\right) r}{\mu} d \tau+k(0) .
\end{aligned}
$$

The MRAC laws of roll and yaw channels can be designed in the same way. However, different reference model for each channel is selected based on the performance index of respective channel. The MRAC system diagram of attitude control system is shown as Figure 2.

Remark 2. The control performance indexes of each channel determine the form of the reference model. Although model uncertainties and gust disturbances exist in the actual system, only if the output of system can track the output of reference model, the performance can be guaranteed. Therefore, MRAC system has strong robustness with respect to the model uncertainties and external disturbances.

4.2. PID Control Law Design. For the simplified model of pitch channel, PID control law can be obtained. The expression of control law is

$$
\bar{u}_{2}=k_{1} \bar{e}+k_{2} \int_{0}^{t} \bar{e} d t+k_{3} \dot{\bar{e}},
$$

where $\bar{e}$ is the error between reference input and system output; Gains $k_{1}, k_{2}$, and $k_{3}$ can be determined by root locus according to the control performance indexes of pitch channel.

In the same way, the control laws of roll and yaw channels can be designed by PID method and the control diagram of attitude control system for UAV is shown as Figure 3. 


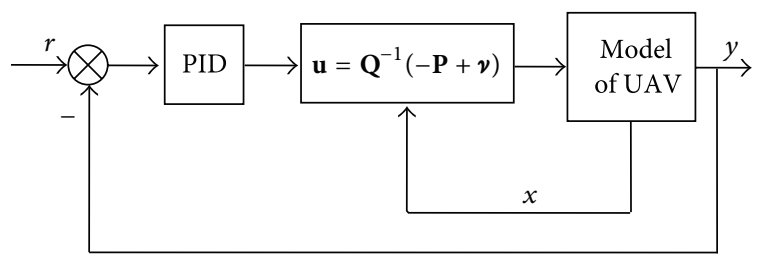

FIGURE 3: PID control system diagram.

\section{Mathematics Simulations}

In order to verify the performance of attitude control system for UAV, the PID control law and the MRAC law are applied to the coupling and nonlinear attitude dynamic model of UAV, respectively.

The reference motion states are as follows:

$$
V=1360 \mathrm{~m} / \mathrm{s}, \quad H=30 \mathrm{Km} \text {. }
$$

The initial conditions of simulation are

$$
\phi=\theta=\psi=0^{\circ}, \quad p=q=r=0 \mathrm{rad} / \mathrm{s} .
$$

The allowed maximum deflection angles of three actuators in simulation are:

$$
\begin{gathered}
-5^{\circ} \leq \delta_{a} \leq 5^{\circ}, \quad-15^{\circ} \leq \delta_{e} \leq 15^{\circ}, \\
-10^{\circ} \leq \delta_{r} \leq 10^{\circ} .
\end{gathered}
$$

The reference inputs of three attitude channels are $10^{\circ}$ step signals. The control performance of attitude control system will be verified through below three cases: Case 1, there is no uncertainty in the system; Case 2, aerodynamic parameters vary within the range of $0 \sim 30 \%$; Case 3 , gust disturbance is considered as the external disturbance.

Figures 4, 5, and 6 show the output responses of roll, pitch, and yaw channels for the above three cases, respectively, wWhere solid line represents the attitude angle under MRAC law and dashed line represents the attitude angle under PID control law.

The performance indexes of attitude control system under all cases are listed in Table 1.

We can see from above that there is almost no difference for attitude angle response under MRAC laws for all cases shown in Figures 4 to 6 . In other words, the control performance indexes still can be satisfied even with parameter perturbation and external disturbance. Adjust PID control law parameters and make the control performance under Case 1 to satisfy the design index. However, the maximum overshoot and settling time of output response will increase while the same PID parameters are applied to Case 2 and Case 3. The control performance becomes worse.

\section{Conclusions}

The design of attitude control system for UAV is presented by integrating feedback linearization and MRAC methods. The complicated coupling nonlinear dynamic model was

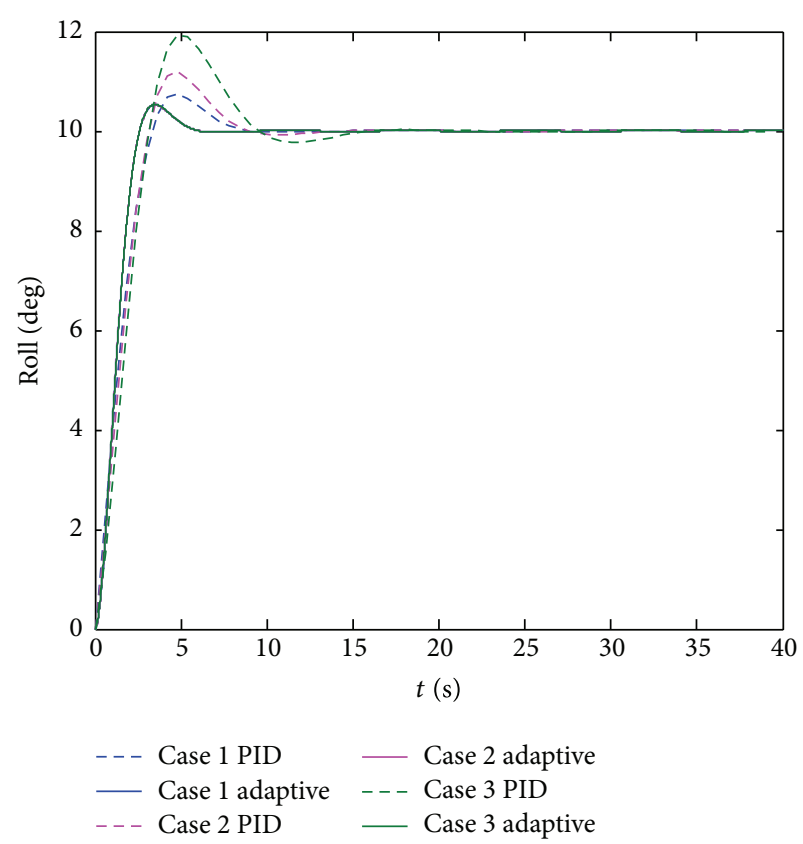

FIGURE 4: Output response of roll channel.

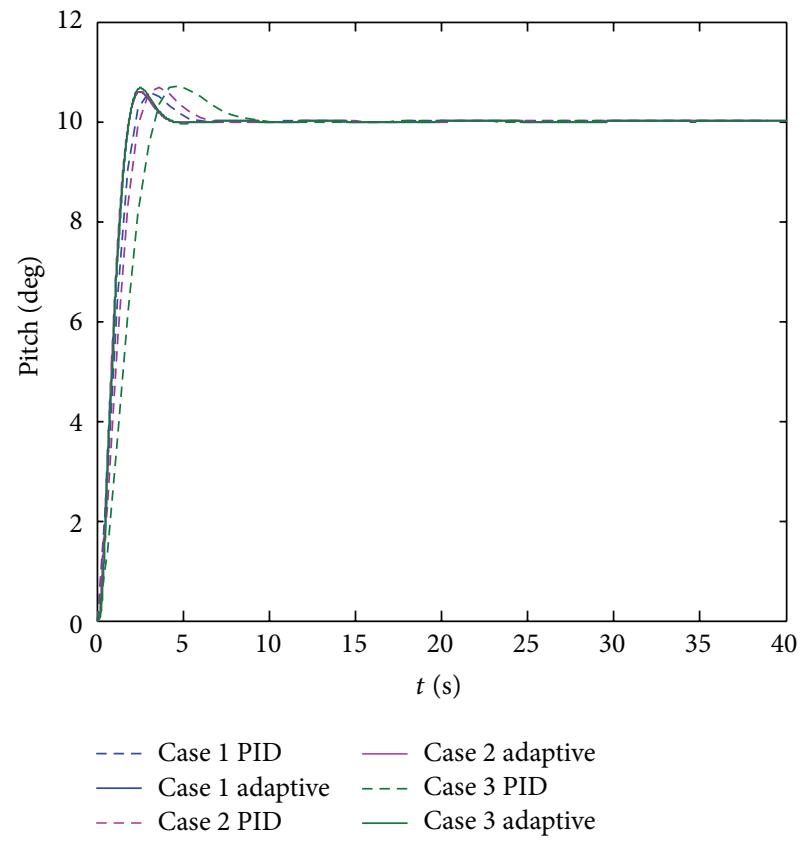

FIGURE 5: Output response of pitch channel.

decoupled into three independent SISO systems by feedback linearization. Then, the control law of each channel was designed using MRAC method and PID method, respectively. The mathematics simulation results indicate that the attitude control system can achieve better control performance including maximum overshoot, settling time, and rise time under MRAC law than that under PID control law. In addition, a stronger robustness with respect to aerodynamic parameter perturbation and gust disturbance has been obtained in MRAC system. 


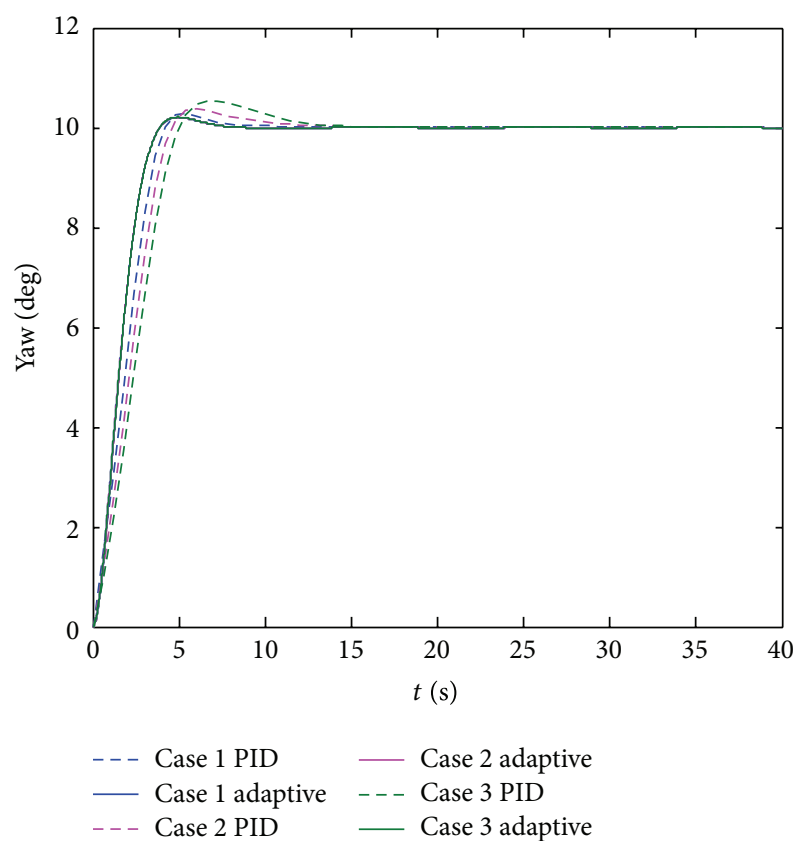

FIGURE 6: Output response of yaw channel.

TABLE 1: Comparison of control performance indexes between MRAC and PID control.

\begin{tabular}{|c|c|c|c|c|}
\hline & Indexes & Case 1 & Case 2 & Case 3 \\
\hline & \multicolumn{4}{|c|}{ Roll channel } \\
\hline \multirow{3}{*}{ PID } & $\delta_{\max }$ & $7.4 \%$ & $11.8 \%$ & $19.2 \%$ \\
\hline & $t_{s}(\mathrm{~s})$ & 7.21 & 7.77 & 12.71 \\
\hline & $t_{r}(\mathrm{~s})$ & 3.13 & 3.14 & 3.34 \\
\hline \multirow{3}{*}{ Adaptive } & $\delta_{\max }$ & $5.2 \%$ & $5.3 \%$ & $5.3 \%$ \\
\hline & $t_{s}(\mathrm{~s})$ & 4.86 & 4.87 & 4.88 \\
\hline & $t_{r}(\mathrm{~s})$ & 2.58 & 2.58 & 2.59 \\
\hline & \multicolumn{4}{|c|}{ Pitch channel } \\
\hline \multirow{4}{*}{ PID } & $\delta_{\max }$ & $5.9 \%$ & $7.0 \%$ & $7.2 \%$ \\
\hline & $t_{s}(\mathrm{~s})$ & 4.62 & 5.35 & 7.46 \\
\hline & $t_{r}(\mathrm{~s})$ & 2.27 & 2.45 & 3.33 \\
\hline & $\delta_{\max }$ & $5.9 \%$ & $6.0 \%$ & $6.7 \%$ \\
\hline \multirow[t]{4}{*}{ Adaptive } & $t_{s}(\mathrm{~s})$ & 3.57 & 3.58 & 3.66 \\
\hline & $t_{r}(\mathrm{~s})$ & 1.85 & 1.85 & 1.87 \\
\hline & \multicolumn{4}{|c|}{ Yaw channel } \\
\hline & $\delta_{\max }$ & $2.8 \%$ & $3.7 \%$ & $5.4 \%$ \\
\hline \multirow[t]{3}{*}{ PID } & $t_{s}(\mathrm{~s})$ & 6.26 & 8.18 & 10.74 \\
\hline & $t_{r}(\mathrm{~s})$ & 4.17 & 4.61 & 5.04 \\
\hline & $\delta_{\max }$ & $2.1 \%$ & $2.1 \%$ & $2.1 \%$ \\
\hline \multirow[t]{2}{*}{ Adaptive } & $t_{s}(\mathrm{~s})$ & 5.27 & 5.32 & 5.35 \\
\hline & $t_{r}(\mathrm{~s})$ & 3.90 & 3.89 & 3.88 \\
\hline
\end{tabular}

\section{Conflict of Interests}

The authors declare that there is no conflict of interests regarding the publication of this paper.

\section{References}

[1] M. Lorenzo, N. Roberto, and I. Alberto, "High-gain output feedback for a miniature UAV," Journal of Robust and Nonlinear Control, 2013.

[2] B. Godbolt, N. I. Vitzilaios, and A. F. Lynch, "Experimental validation of a helicopter autopilot design using model-based PID control," Journal of Intelligent and Robotic Systems, vol. 70, pp. 385-399, 2013.

[3] H. Chao, Y. Luo, L. Di, and Y. Q. Chen, "Roll-channel fractional order controller design for a small fixed-wing unmanned aerial vehicle," Control Engineering Practice, vol. 18, no. 7, pp. 761-772, 2010.

[4] T. Yamasaki, S. N. Balakrishnan, and H. Takano, "Integrated guidance and autopilot design for a chasing UAV via high-order sliding modes," Journal of the Franklin Institute, vol. 349, no. 2, pp. 531-558, 2012.

[5] A. R. Babaei, M. Mortazavi, and M. H. Moradi, "Classical and fuzzy-genetic autopilot design for unmanned aerial vehicles," Applied Soft Computing Journal, vol. 11, no. 1, pp. 365-372, 2011.

[6] B. T. Whitehead and S. R. Bieniawski, "Model reference adaptive control of a quadrotor UAV," in Proceedings of the AIAA Guidance, Navigation, and Control Conference, Toronto, Canada, 2010.

[7] S. Kurnaz, O. Cetin, and O. Kaynak, "Adaptive neuro-fuzzy inference system based autonomous flight control of unmanned air vehicles," Expert Systems with Applications, vol. 37, no. 2, pp. 1229-1234, 2010.

[8] E. Capello, G. Guglieri, F. Quagliotti, and D. Sartori, "Design and validation of an L1 adaptive controller for mini-UAV autopilot," Journal of Intelligent and Robotic Systems, vol. 69, pp. 109-118, 2013.

[9] A. R. Babaei, M. Mortazavi, and M. H. Moradi, "Fuzzy sliding mode autopilot design for nonminimum phase and nonlinear UAV," Journal of Intelligent and Fuzzy Systems, vol. 24, pp. 499509, 2013.

[10] H. Pu, Z. Zhen, and D. Wang, "Modified shuffled frog leaping algorithm for optimization of UAV flight controller," International Journal of Intelligent Computing and Cybernetics, vol. 4, no. 1, pp. 25-39, 2011.

[11] H. Z. Pu, Z. Y. Zhen, J. Jiang, and D. B. Wang, "UAV flight control system based on an intelligent BEL algorithm," Journal of Advanced Robotic Systems, vol. 10, no. 121, pp. 1-8, 2013.

[12] C. X. Lu and Q. Huang, "Design of the platform for a UAV flight control system based on STM32," Journal of Digital Content Technology and Its Applications, vol. 7, no. 5, pp. 1033-1041, 2013.

[13] I. Yavrucuk and V. Kargin, "Autolanding controller strategies for a fixed wing UAV in adverse atmospheric conditions," in Proceedings of the AIAA Guidance, Navigation and Control Conference and Exhibit, Portland, Ore, USA, August 2008.

[14] Y. C. Paw and G. J. Balas, "Development and application of an integrated framework for small UAV flight control development," Mechatronics, vol. 21, no. 5, pp. 789-802, 2011.

[15] A. B. Milhim and Y. M. Zhang, "Quad-rotor UAV: highfidelity modeling and nonlinear PID control," in Proceedings of the AIAA Modeling and Simulation Technologies Conference, Toronto, 2010

[16] M. Lungu and R. Lungu, "Adaptive backstepping flight control for a mini-UAV," International Journal of Adaptive Control and Signal Processing, vol. 27, no. 8, pp. 635-650, 2013. 
[17] J. Shin, H. Jin Kim, and Y. Kim, "Adaptive support vector regression for UAV flight control," Neural Networks, vol. 24, no. 1, pp. 109-120, 2011.

[18] H. Gao, T. Chen, and J. Lam, "A new delay system approach to network-based control," Automatica, vol. 44, no. 1, pp. 39-52, 2008.

[19] H. Yan, Z. Su, H. Zhang, and F. Yang, "Observer-based $H_{\infty}$ control for discrete-time stochastic systems with quantisation and random communication delays," IET Control Theory \& Applications, vol. 7, no. 3, pp. 372-379, 2013.

[20] H. Zhang, H. Yan, F. Yang, and Q. Chen, "Distributed average filtering for sensor networks with sensor saturation," IET Control Theory \& Applications, vol. 7, no. 6, pp. 887-893, 2013.

[21] S. Y. Yang, S. J. Tang, C. Liu, and J. Guo, "Design and verification the simulation system of UAS flight control considering the effects of time delay," Applied Mechanics and Materials, vol. 278280, pp. 1746-1753, 2013.

[22] H. Zhang, H. Yan, F. Yang, and Q. Chen, "Quantized control design for impulsive fuzzy networked systems," IEEE Transactions on Fuzzy Systems, vol. 19, no. 6, pp. 1153-1162, 2011.

[23] L. X. Zhang, H. J. Gao, and O. Kaynak, "Network-induced constraints in networked control systems-a survey," IEEE Transactions on Industrial Informatics, vol. 9, no. 1, pp. 403-416, 2013.

[24] J.-P. Gao and Z.-J. Chen, "Study on gain-scheduling problem in flight control," Chinese Journal of Aeronautics, vol. 12, no. 4, pp. 217-221, 1999. 


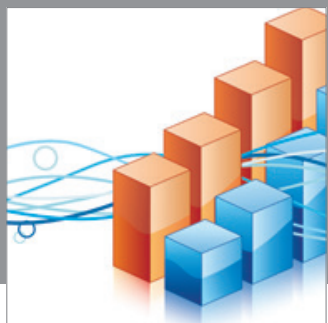

Advances in

Operations Research

mansans

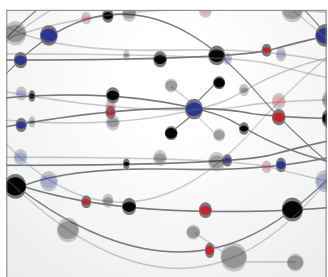

The Scientific World Journal
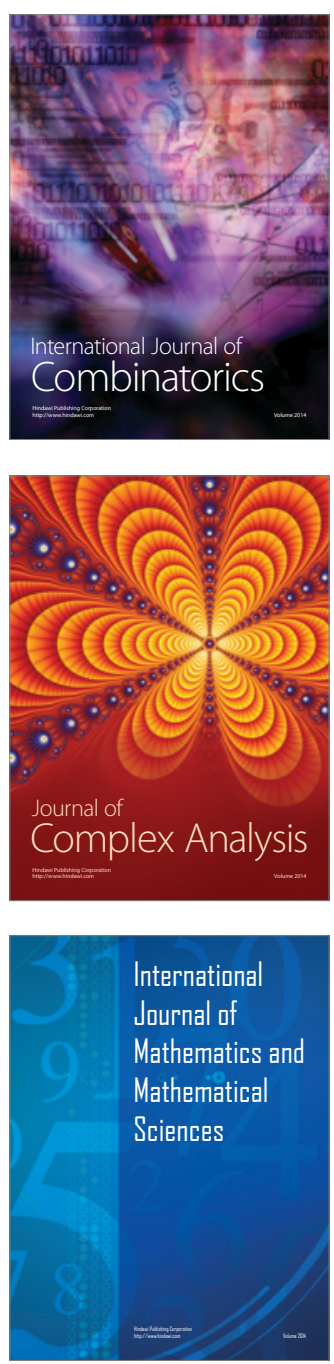
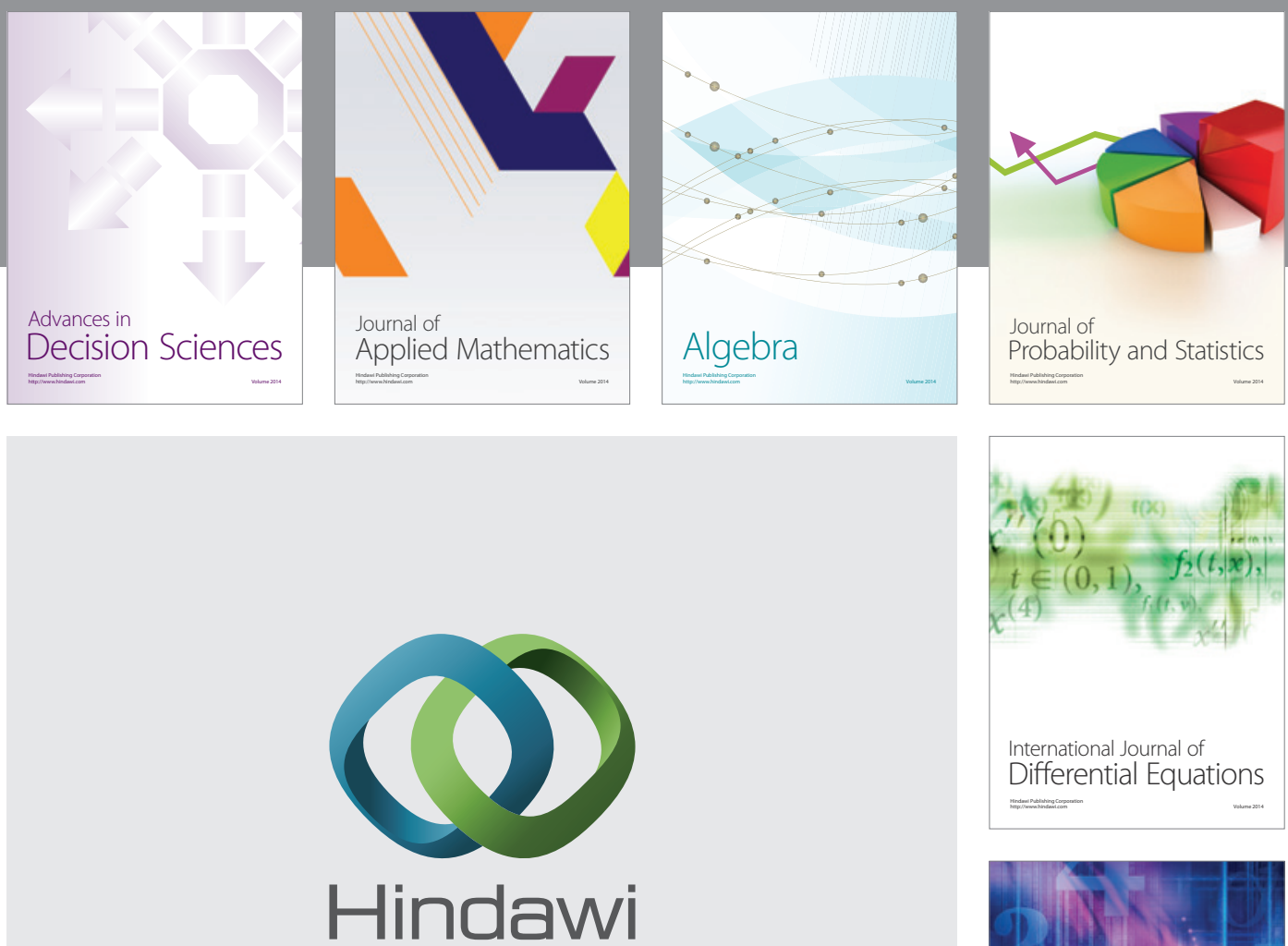

Submit your manuscripts at http://www.hindawi.com
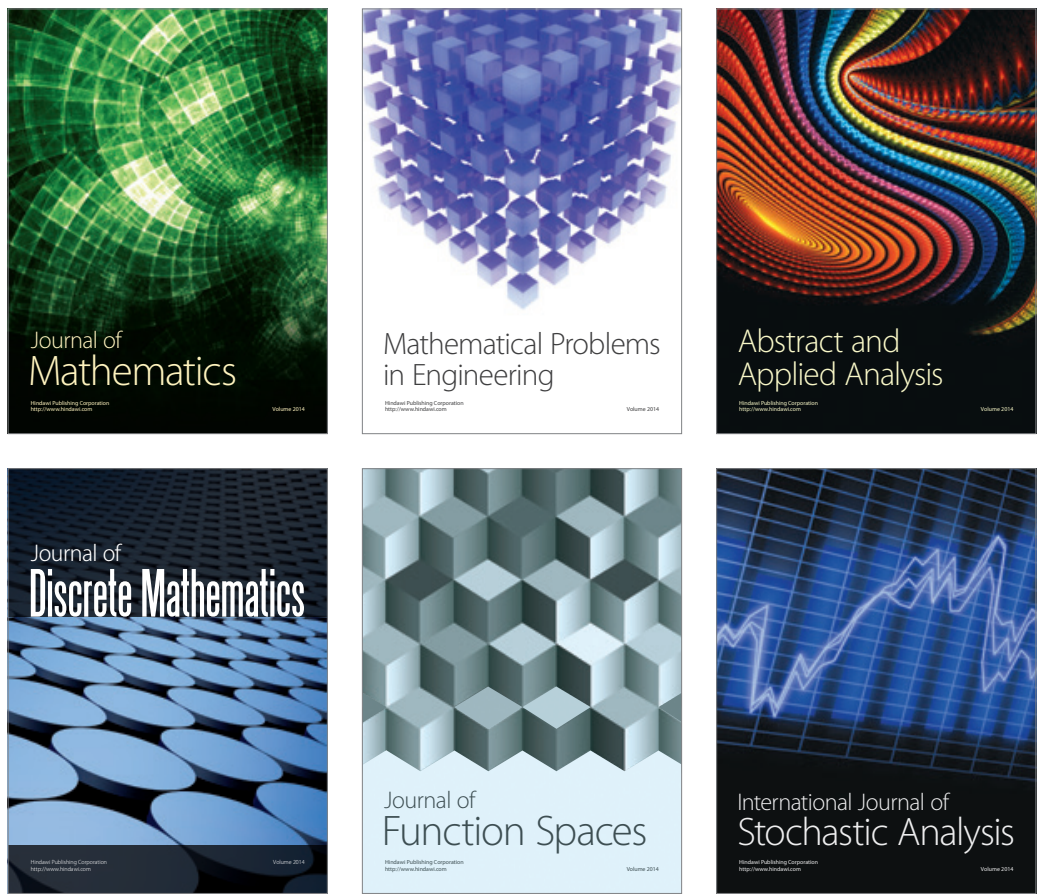

Journal of

Function Spaces

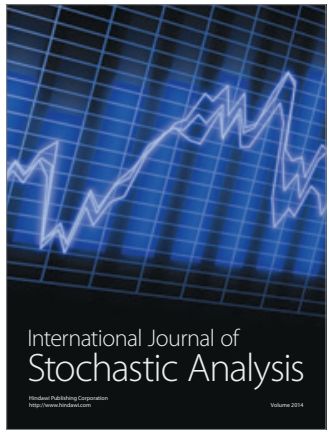

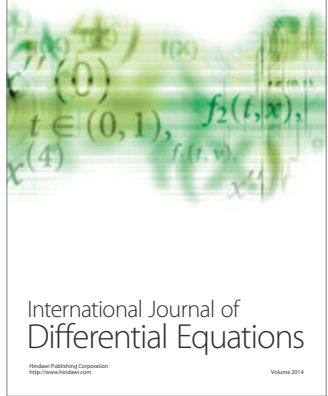
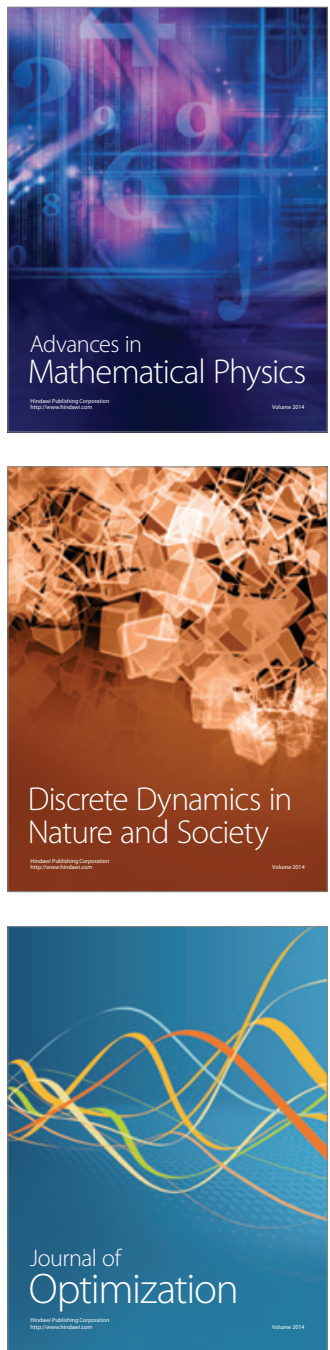